\title{
A Novel Technique for Synthetic Source of Multiple Electromagnetic Data Sets*
}

\author{
Liting Rao*, Xin Wu, Rui Guo, Pan Xiao, Xiaojuan Zhang, Guangyou Fang \\ Key Laboratory of Electromagnetic Radiation and Sensing Technology \\ Chinese Academy of Sciences \\ Beijing, China \\ raoliting11@mails.ucas.ac.cn
}

\begin{abstract}
A novel technique for synthetic source of multiple electromagnetic data sets is presented. The technique applies weighting to multiple transmitters of small magnetic moment loops to form a synthetic source for deep deposits exploration in complex terrain. In order to determine the weighting coefficient, a dipole model is employed to consider the coupling between the target and each transmitter and receiver. By assuming the possible orientation and location of the target, a look-up table of transmitting and receiving coupling coefficient is established to search for the approximate location and orientation of the target. Once the location and orientation of the target is decided through the matching process, a composite transmitter can be formed by summing the single transmitter data using weights calculated based on the coupling between the target and the transmitters, then the response from that target will be maximally enhanced.
\end{abstract}

Keywords - synthetic source; dipole model; look-up table; deep deposits exploration

\section{INTRODUCTION}

With the increasing exploitation of mineral resources, exploration for deeply concealed deposits in complex terrain will be essential in sustaining current mine production levels and future demand for resources. The transient electromagnetic method (TEM) is widely used in groundwater and environmental investigations, as well as being an exploration tool for mineral resources ${ }^{[1]}$. It is based on the principle of electromagnetic induction, in which, a transmitter loop on the ground surface is energized by short pulses of direct current. When the current is turned off, the primary field rapidly disappears and the secondary field generated by eddy currents in subsurface conductors can be detected ${ }^{[2-3]}$. In order to deeper soundings, the main approach to meet this challenge is to use large high powered transmitter with large magnetic moment. Because of the limited transmitter current intensity, another option in amplifying magnetic moment can be achieved by increasing the loop area. However, using larger transmitter wire loop is often costly and also impractical in complex terrain. Furthermore, when the transmitter loop is not positioned properly, such that the primary field excitated by the transmitter does not couple well with the target, then the secondary field of the target generated by eddy current may be small enough to be undetectable ${ }^{[4]}$. According to the above reasons, to enhance deeper soundings effectively requires a new methodology. Rather than using large magnetic moment loop, many smaller moment loops can be used and through optimized weighting turn into larger synthetic source ${ }^{[5-7]}$. The goal of synthetic source to multiple electromagnetic data sets is to increase the detectability of deep reservoir and enhance the response from a specific target.

\section{THEORY}

Fig.1 displays the geometry of a multiple transmitter and receiver system for target detection. To ensure the synthetic source highlights the response of a specific target, we use a dipole approximation which assuming the target, transmitters, and receivers as dipoles to solve for the weights used to sum the transmitters. The dipole approximation can efficiently calculate the coupling between the target and transmitter and receiver. The coupling coefficient between the transmitter and the target is obtained by using the formula of primary field at the target location motivated by a dipole source at transmitter position $^{[8]}$. On the similar principle, the coupling coefficient between the receiver and the target can be also acquired. Each coupling coefficient is written as:

$$
\begin{aligned}
& T_{c}\left(\boldsymbol{r}_{t}, \boldsymbol{r}_{d}, \hat{d}_{\theta}\right)=\frac{1}{4 \pi r_{t d}^{3}}\left[3\left(\hat{m}_{t} \cdot \hat{r}_{t d}\right) \hat{r}_{t d}-\hat{m}_{t}\right] \hat{d}_{\theta} \\
& R_{c}\left(\boldsymbol{r}_{s}, \boldsymbol{r}_{d}, \hat{d}_{\theta}\right)=\frac{1}{4 \pi r_{d s}^{3}}\left[3\left(\hat{d}_{\theta} \cdot \hat{r}_{d s}\right) \hat{r}_{d s}-\hat{d}_{\theta}\right] \hat{m}_{r}
\end{aligned}
$$

Where $T_{c}$ is the coupling coefficient between a transmitter located at $\boldsymbol{r}_{t}$ and a dipole target of orientation $\hat{d}_{\theta}$ located at $\boldsymbol{r}_{d}$, $\boldsymbol{R}_{c}$ is the coupling coefficient between a receiver located at $\boldsymbol{r}_{s}$ and a dipole target of orientation $\hat{d}_{\theta}$ located at $\boldsymbol{r}_{d}, \hat{m}_{t}$ and $\hat{m}_{r}$ represent unit direction vector of transmitting dipole and 


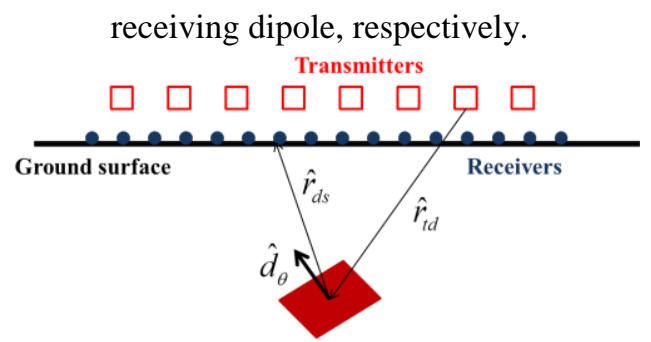

Fig. 1. The geometry of a multiple transmitter and receivers system for target detection

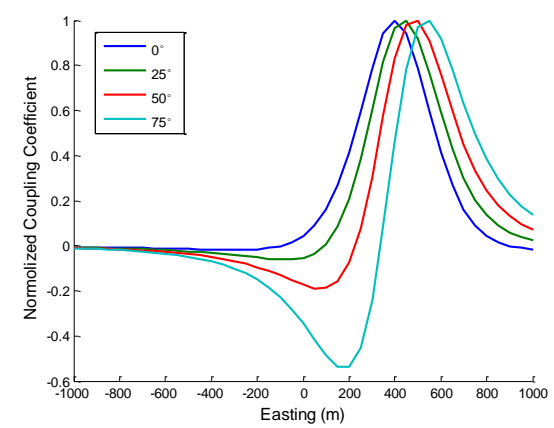

Fig. 2. Normalized coupling coefficient calculated between a dipole target with varying dip and transmitter with varying location

Fig.2 shows the normalized coupling coefficient for a target with varying dip located at $(400 \mathrm{E}, 0 \mathrm{~N},-350 \mathrm{Z})$ and vertical dipole transmitters which is spaced $100 \mathrm{~m}$ apart. As can be seen, the weights vary for different target locations and orientations. The normalized coefficients are the weighting factors applied to the corresponding data related with the transmitter at that location. In this case, the data related with transmitters coupling well to the target are enhanced and those not coupling well are reduced. But the weight factors are determined by the specific target location and orientation which is unknown.

To enhance the response, the target orientation and location must be known. The proposed method is to obtain the target's parameters through matching the look-up table. The look-up table is established by assuming every possible target location and orientation and calculates the coupling coefficient with each transmitter and receiver. Matching degree is defined as follow:

$$
P\left(\boldsymbol{r}_{d}, \hat{d}_{\theta}\right)=\left\{1-\sum \frac{\left[D^{S} \boldsymbol{T}_{c}\left(\boldsymbol{r}_{d}, \hat{d}_{\theta}\right)-\boldsymbol{R}_{c}\left(\boldsymbol{r}_{d}, \hat{d}_{\theta}\right)\right]^{2}}{\boldsymbol{R}_{c}\left(\boldsymbol{r}_{d}, \hat{d}_{\theta}\right)^{2}}\right\}
$$

Where $P\left(\boldsymbol{r}_{d}, \hat{d}_{\theta}\right)$ is the matching degree, $D^{S}$ is the matrix of survey data, where each column represents the data at different receivers and each row represents the data at different transmitters, $\boldsymbol{T}_{c}$ is the vector of coupling coefficients for each transmitter position, and $\boldsymbol{R}_{c}$ is the vector of coupling coefficients for each receiver position. Note that $\boldsymbol{D}^{S}, \boldsymbol{R}_{c}$ and $\boldsymbol{T}_{c}$ are normalized to unit amplitudes. When the assumed location and orientation match the actual location and orientation of the target, matching degree will approach to unity. That's because the survey data can be expressed as the product of the vector of transmitter and receiver coupling coefficients calculated for the actual location and orientation of the target:

$$
D^{S}=R_{c}\left(\boldsymbol{r}_{d}^{0}, \hat{d}_{\theta}^{0}\right) \cdot T_{c}\left(\boldsymbol{r}_{d}^{0}, \hat{d}_{\theta}^{0}\right)^{T}
$$

Where $\boldsymbol{r}_{d}^{0}$ and $\hat{d}_{\theta}^{0}$ represent the actual location and orientation of the target respectively. In this paper, the value of matching degree will be set to zero when the actual value is negative.

\section{EXAMPLE}

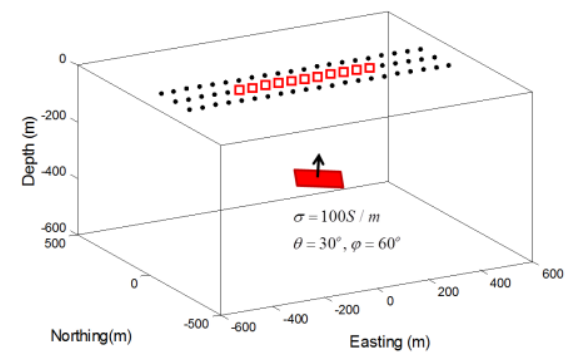

Fig. 3. 3D geometry of model for simulated calculation

The example model is depicted in Fig.3. It shows a $200 \mathrm{~m}$ (strike length) $\times 150 \mathrm{~m}$ (dip length) plate with conductivity of $100 \mathrm{~s} / \mathrm{m}$. The center of the plate is located at $(0,0,-600)$.The plate has a strike $(\varphi)$ of $60^{\circ}$ and a dip $(\theta)$ of $30^{\circ}$. The transmitter loop with side length of $10 \mathrm{~m}$ is spaced every $50 \mathrm{~m}$ from $-250 \mathrm{E}$ to $250 \mathrm{E}$ at $0 \mathrm{~N}$, occupied the inner $500 \mathrm{~m}$ of the line for a total 11 transmitter positions. There are three survey lines for Northing from $-100 \mathrm{~N}$ to $100 \mathrm{~N}$, separated by $100 \mathrm{~m}$. Each line sets 21 receivers from $-500 \mathrm{E}$ to $500 \mathrm{E}$.

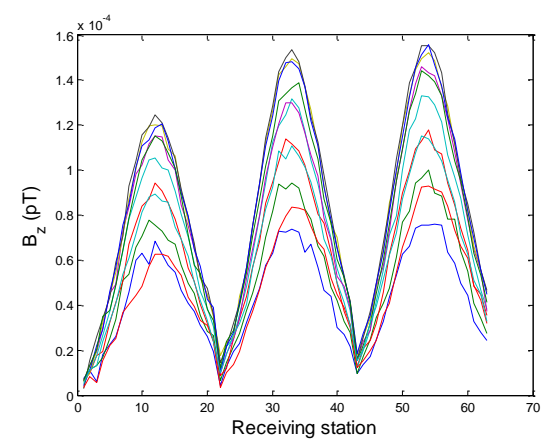

Fig. 4. Data from each receiving station with adding 5\% random Gaussian noise 
In order to simulate the measurement data, 5\% random Gaussian noise was added to the synthetic data. The Fig.4 shows, survey data used to determine the target location and orientation. In this example, the strike of the dipole varied from $0^{\circ}$ to $90^{\circ}$ in $10^{\circ}$ intervals, the dip of the dipole varied from $0^{\circ}$ to $350^{\circ}$ in $10^{\circ}$ intervals. The possible dipole area is set from $-500 \mathrm{E}$ to $500 \mathrm{E},-500 \mathrm{~N}$ to $500 \mathrm{~N}$, and $-800 \mathrm{Z}$ to $-400 \mathrm{Z}$, and the discretized subsurface cell size is $50 \mathrm{~m}$.

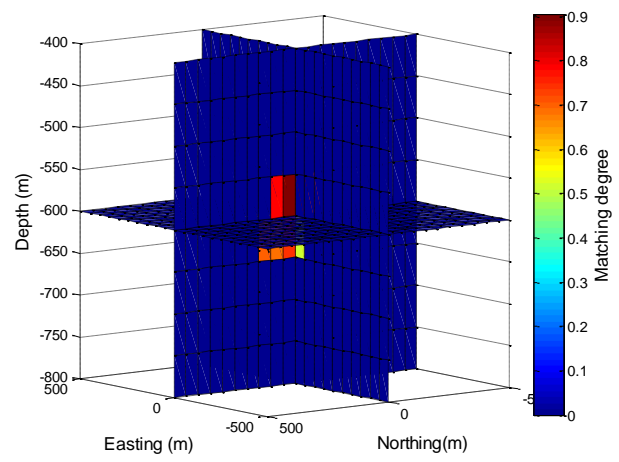

Fig. 5. Three-dimensional slice map of matching degree.

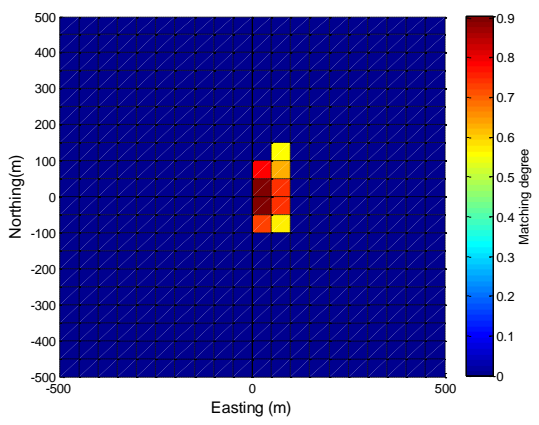

(a)

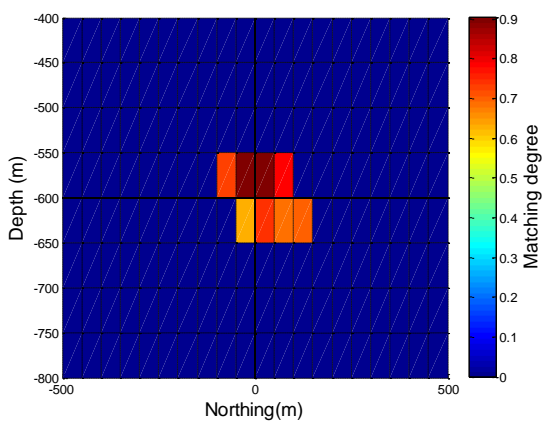

(b)

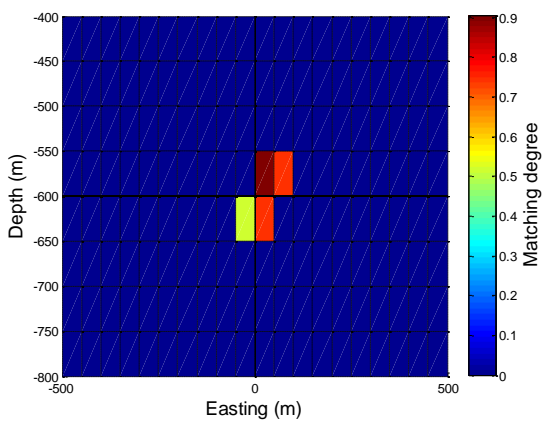

(c)

Fig. 6. (a) Horizontal slice view at depth=-600 m, (b) Vertical slice view at easting $=0 \mathrm{~m}$, (c) Vertical slice view at northing $=0 \mathrm{~m}$.

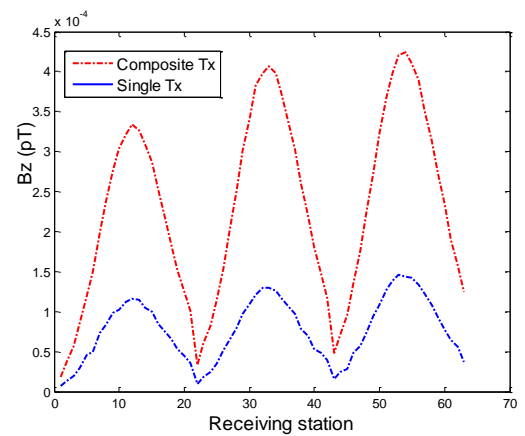

Fig. 7. Comparison of data from the summed composite transmitter (dashed black line) and the single transmitter (solid blue line)

Fig.5 shows the three-dimensional slice map of matching degree which represents maximum $\mathrm{P}$ value of all possible orientation at the corresponding position. When matching degree was less than 0.5 , the color was set to blue. It's clear that most of the $\mathrm{P}$ values greater than 0.5 focuses on the actual target location. As can be seen in Figure 6, the location of the maximum $\mathrm{P}$ value matches the actual location. The matching degree that produced the maximum $\mathrm{P}$ value corresponded to a dipole located at $(0 \mathrm{~N}, 0 \mathrm{E}$, and $-600 \mathrm{Z})$ with a strike and dip of $30^{\circ}$ and $60^{\circ}$, respectively. This clearly indicates that the dipole model is an effective approximation for determining the location and orientation of the target embedded in a resistive surrounding.

Once the location and orientation of the target can be determined through the maximum $\mathrm{P}$ value, the multiple transmitter data can be summed to form a composite transmitter:

$$
\boldsymbol{D}^{c}=\boldsymbol{D}^{s} \boldsymbol{T}_{\boldsymbol{c}}\left(\boldsymbol{r}_{d}, \hat{d}_{\theta}\right)
$$

A comparison of the data from the composite transmitter and a single transmitter is showed in Fig.7. Note the amplitude of the composite transmitter is roughly 3 times larger than that of a single transmitter data. 


\section{CONCLUSIONS}

This paper presents a novel technique for deep mineral deposits exploration, which will be required in a complex terrain. Rather than using large magnetic moment loop, many smaller moment loops can be used and through optimized weighting turn into larger synthetic source. The weighting coefficients are related to the target location and orientation. The approximate location and orientation can be found by comparing the measured response with the coupling look-up table which is established by discretizing the subsurface and possible target orientations. Through a synthetic example, it is clear that the dipole model is an adequate approximation for determining the location and orientation of target embedded in a resistive surrounding. At last the multiple transmitter data can be summed to form a high $\mathrm{S} / \mathrm{N}$ composite transmitter.

\section{REFERENCES}

[1] G. Q. Xue, C. Y. Bai, et al., "Deep sounding TEM investigation method based on a modified fixed central-loop system," Journal of Applied Geophysics, 2012, 76, pp. 23-32.
[2] F. S. Grant, and G. F. West, Interpretation theory in applied geophysics: McGraw-Hill, 1965.

[3] M. N. Nabighian, et al., "Electromagnetic methods in applied geophysics-Theory Volume I," Society of Exploration Geophysicists, 1988, pp. 313 503.

[4] J. Lymburner, R. S. Smith, "A procedure for collecting electromagnetic data using multiple transmitters and receivers capable of deep and focused exploration," Geophysics, 2015, 80, pp. E1-E10.

[5] Zhdanov, M.S., 2010, Electromagnetic geophysics: Notes from the past and the road ahead: Geophysics, 75, 75A49-75A66.

[6] Zhang, J. S., Wu X., et al., 2013, Research on 3D marine electromagnetic interferometry with synthetic sources for suppressing the airwave interference: Applied Geophysics, 10, 373-383.

[7] Knaak A., Snieder R., et al., 2015, Optimized 3D synthetic aperture for controlled-source electromagnetics: Geophysics, 80, E309-E316.

[8] Smith R. S., Lee T. J., 2001, The impulse-response moments of a conductive sphere in a uniform field, a versatile and efficient electromagnetic model: Exploration Geophysics, 32, 113-118. 